\title{
Bible, poésie et doctrine dans la Josephina de Jean Gerson
}

Connu surtout comme théoricien de la théologie mystique et par son action réformatrice en tant que Chancelier de l'Université de Paris au début du XVe siècle, Jean Gerson nous a aussi légué une œuvre poétique latine d'envergure ${ }^{1}$. Loin d'être un exercice marginal, celle-ci, comme le fait remarquer G. Matteo Roccati, «trouve sa place dans la logique de l'ensemble de sa production et elle en partage thèmes et préoccupations ${ }^{2}$.» C'est le cas notamment de la Josephina, poème épique de près de 3000 hexamètres, composé entre 1414 et $1417^{3}$, pour l'essentiel au concile de Constance. À travers le récit de l'histoire de la Sainte Famille depuis l'Annonciation jusqu'à la mort de Joseph, Gerson entend promouvoir le culte du mariage virginal des saints époux comme exemple de l'union du Christ et de l'Église dans un contexte de trouble politique et ecclésial ${ }^{4}$.

La Josephina puise une bonne partie de son contenu dans les Évangiles, l'Ancien Testament, et, indirectement, dans les apocryphes ${ }^{5}$. Elle comporte aussi de nombreuses réminiscences classiques. À cet égard, le choix de forme métrique n'est pas anodin. Traditionnellement réservé à l'épopée antique, l'hexamètre dactylique connaît un nouvel essor au tournant du $\mathrm{Xv}^{\mathrm{e}}$ siècle grâce à l'Africa de Pétrarque. L'affinité entre les deux épopées ne relève pas d'un simple aspect formel. Tout comme l'Africa, la Josephina comporte une dimension de fierté nationale, où l'auteur entend s'ériger en poète de la res gallica ${ }^{6}$. Il ne s'agit pas ici de célébrer les exploits guerriers d'un Scipion ou d'un Enée en vue de faire reconnaître la suprématie romaine, mais de chanter la primauté intellectuelle de Paris injustement décriée par Pétrarque.

Mais malgré toutes ses affinités avec la production littéraire des italiens de la période $^{7}$, la Josephina ne pointe vers aucune source précise qui pourrait nous guider

1 Voir Roccati (1980) ; Roccati (2001) ; Glorieux (1962). Ici, les références à l'édition critique de la Josephina et à sa traduction française renvoient toutes à Iribarren- Roccati (2018).

2 Roccati (2001) 5. Voir aussi Hobbins (2009) 92-101.

3 Au sujet de la datation du poème, voir Roccati (2001) 15-24; Lieberman (1955) 289-333, surtout 323-326 ; Combes (1964) 290-297; Glorieux (1962) 15.

4 Pour l'histoire du culte de saint Joseph, l'ouvrage de référence reste Seitz (1908) ; voir aussi Dordoni (1996) 321-342.

5 Au sujet de l'utilisation des apocryphes dans la Josephina, je me permets de renvoyer le lecteur à Iribarren (2018).

6 On retrouve les mêmes idées dans le traité inachevé contre Juan de Monzón, où le jeune Gerson, alors bachelier à Paris, déplore que la France, pourtant si riche en hommes sages, manque toujours d'historiens et de poètes pour les célébrer. Voir Ouy (1962) 433-492, en part. p. 447-450; voir aussi Fabre (2006) 111-125.

7 À d'autres occasions, Gerson exprime aussi la volonté de s'exercer dans des genres nouveaux, comme les mètres utilisés par Boèce. Voir à ce sujet Burrows (1991). 
vers un modèle éventuel ${ }^{8}$. Pour décrire son poème, Gerson utilise des termes d'origines variées. Dans le Prologue (v. 92), il emploie l'antique camena ; ailleurs, il emprunte des termes de la tradition classique, comme carmen (Prol., v. 71, 83 et 99 ; dist. I, v. 272, 371 ; dist. II, v. 728 ; dist. III, v. 1007) ou oda (dist. I, v. 274) ; il recourt aussi au registre de la poésie liturgique comme le verbe psallere et ses dérivés (dist. I, v. 274 ou dist. IV, v. 982). Dans la composition du poème, l'auteur agence soigneusement divers types de registres : les passages narratifs alternent avec des descriptions, des dialogues «dramatisés» et des réflexions théologiques. On y trouve également des exercices plus traditionnels de la paraphrase biblique des passages les plus connus des évangiles : le Magnificat (v. 1595-1620), le Benedictus (v. 1735-1753), le Nunc dimittis (v. 2369-2374).

Trois aspects de l'épopée, sur lesquels on s’attardera tour à tour, font ressortir la conception gersonienne du rapport entre Bible, poésie et développement doctrinal : d'abord, son organisation formelle en distinctiones ; deuxièmement, la notion d'«estimation pieuse» qui gouverne la composition ; enfin, les présupposés herméneutiques de l'affaire Jean Petit sur le tyrannicide.

\section{Organisation formelle du poème}

Certains aspects de l'organisation formelle du texte se conforment à un genre bien défini. Il est constitué d'un Exordium, d'un résumé (Titulus brevius) et de douze distinctiones, dont la douzième s'achève par une Oratio finale. À l'instar de l'Énéide, le modèle du genre, Gerson veut manifestement se conformer à la répartition classique en douze chants, même si, à la manière scolastique, il utilise le terme de distinctio pour les désigner. Ce choix est significatif et mérite un commentaire?

Évocatrices de la culture d'école, les distinctiones renvoient tout d'abord à une technique bien attestée depuis le $\mathrm{XII}^{\mathrm{e}}$ siècle chez les exégètes, et adoptée massivement par les prédicateurs dès le $\mathrm{XIII}^{\mathrm{e}}$ siècle. Dans sa définition la plus générale, la distinctio consiste dans le déploiement des diverses significations figurées ou symboliques d'un terme ou d'un passage biblique, souvent tempérées par le recours à des exempla. À la fin du XII ${ }^{\mathrm{e}}$ siècle, on assiste à la rédaction des premiers recueils de distinctiones, sortes de classements ordonnés alphabétiquement des sens multiples des mots bibliques ${ }^{10}$. Gerson hérite de cette tradition et la met à profit dans la Josephina. Selon le chancelier, les distinctiones désignent une forme d'organisation

8 Voir Roccati (1982) 278-285.

9 Pour une typologie des formes de l'exégèse médiévale, voir Dahan (1999) 121-159.

10 Leur utilité comme instruments de travail dans le cadre de la prédication est bien attestée. Pour des études générales sur les distinctiones bibliques et leur impact sur la prédication, voir Rouse (1974) 27-37 ; Bataillon (1982) 213-226 ; Bataillon (1993) 280 - 289 ; Weijers (1991) 120-126 ; Dahan (1999) 131-135; 134-138 et 145-151 ; Hasenohr (1978) 46-96 et 183-206 ; Wilmart (1940) 307-346 ; Landgraf (1973) 57-58. Chenu (1964) 129-135, surtout 130-131. 
du discours dont la vertu formelle doit servir l'affect : «de façon succincte elles [les distinctiones] éclairent l'intellect et sans trop de peine charment l'affect ; c'est ainsi que la parole vive et efficace de Dieu pénètre doucement, à la manière d'une conversation, par moyen de parties ordonnées (per particulas ordinatim) ${ }^{11}{ }^{\prime}$. Davantage que le caractère succinct de la distinctio, ce qui doit retenir notre attention dans le passage cité est l'adverbe ordinatim : les distinctions permettent la présentation ordonnée d'un savoir doctrinal, visant en l'occurrence la progression vers l'union à Dieu par une voie affective. Elles forment un tout organique, permettant de passer par étapes des parties les plus élémentaires d'un verset biblique à la construction d'un discours plus complexe où ressort la diversité de ses sens spirituels. La distinction est ainsi le support d'une mise en ordre du savoir employée emblématiquement dans les sommes et commentaires des Sentences scolastiques ${ }^{12}$, et qui dans la Josephina est fondé sur un itinéraire spirituel et non chronologique. En effet, à l'instar de l'ordo artificialis de l'Enéide, la trame narrative de la Josephina ne respecte pas l'ordre chronologique des cycles de l'Enfance, qui débutent d'ordinaire avec l'Annonciation, mais choisit de commencer par la fuite en Égypte. Le choix opéré par Gerson fait non seulement ressortir l'aspect dramatique de la vie du protagoniste ${ }^{13}$, mais favorise une lecture anagogique qui met en exergue l'ascension mystique de l'âme, thème qui sous-tend le récit. En effet, comme l'a montré Isabelle Fabre, le lien fonctionnel entre poésie et mystique constitue la base de la réhabilitation théologique de l'inspiration poétique chez Gerson ${ }^{14}$. Dans ce but, il mobilise les topoi traditionnels : le contraste entre les Muses de la poésie profane et le Christ comme source d'inspiration de la poésie chrétienne (Prologue) ${ }^{15}$; le recours aux modèles bibliques extraits des commentaires des Psaumes, notamment la figure de David «le

11 Gerson, Gratia tibi, lettre à Michel Bartine, moine de la Grande Chartreuse, Lyon, 9 juin 1426, éd. P. Glorieux, Euvres complètes, vol. II.276 [désormais éd. Glorieux, vol. et p.] : sermones magistrales vel determinationes in materiis particularibus qui fiunt succinte per distinctiones, valde illuminant intellectum et sine labore gravi demulcent etiam affectum, cum inspicitur sermo Dei vivus et efficax dum ad modum colloquentis eidem se infundit suaviter per particulas ordinatim.

12 Dans la scolastique, le terme ordinatio désigne le plus souvent le principe qui guide la mise en ordre d'une matière donnée, dans une logique de subordination des savoirs aux Écritures. Voir par exemple Hugues de Saint-Victor, De sacramentis, Prol., c. 6 (PL 176), col. 185 : omnes artes naturales divinae scientiae famulantur, et inferior sapientiae recte ordinata ad superiorem conducit ; Bonaventure, Collationes in Haexaemeron, XIX.6, dans Opera omnia V, Quaracchi, 421 ; Vincent de Beauvais, Libellus totius operis apologeticus, Prologue au Speculum maius, c. 2, in : S. Lusignan (éd.) (1979), Préface au "Speculum majus» de Vincent de Beauvais : réfraction et diffraction, Montréal-Paris, 117. À ce sujet, voir Parkes (1976) 115-141.

13 Voir Roccati (2001) 11. Sur l'ordo artificialis dans la poésie médiévale, voir Zink (2003) 95-97, surtout 97 n. 1.

14 Voir Fabre (2011) 79-107. Voir aussi Witt (1977) 538-563.

15 Gerson, Prebe fidem, in : Fabre (2005) 458, v. 51-54 et 457, v. 17-24 ; Gerson, De laudibus elegie spiritualis (Quisquis amas), in : Fabre (2005) 455, v. 5-8; Gerson, De laude musice (Musica divini), in : Fabre (2005) 449-450, v. 43-44; Gerson, Tractatus de canticis, in : Fabre (2005) 151 et 322 . À ce sujet, voir aussi Zink (2003) 110-111. 
berger citharède ${ }^{16}$; enfin, l'emphase sur la dimension morale et pédagogique de la poésie ${ }^{17}$.

Comme le veut la pratique universitaire de la distinctio biblique, la démarche de Gerson réserve toute sa place aux techniques de communication de la doctrine, que les artes praedicandi des siècles antérieurs avaient résumée dans la trilogie auctoritates - rationes - exempla ${ }^{18}$. L'application de ces pratiques dans la Josephina nous fait comprendre que les rationes tiennent moins ici d'une dialectique universitaire appliquée aux concepts ${ }^{19}$ que de l'exercice d'amplification rhétorique décrite par Cicéron dans le De Inventione ${ }^{20}$ et dans la Rhetorica ad Herennium, textes qui ont connu une large diffusion dans les écoles depuis au moins le $\mathrm{xI}^{\mathrm{e}}$ siècle ${ }^{21}$. La Rhetorica ad Herennium emploie le terme de argumentum à cet égard comme un type de narration mitoyenne entre la historia, qui relate les choses réellement survenues, et la fabula, qui porte sur l'invraisemblable : l'argumentum porte sur les «choses qui auraient pu arriver» mais qui ne sont pas réellement arrivées. Ce genre de récit cherche le veri similis, selon ce qui est «habituel, attendu et naturel ${ }^{22}$.

\section{«Estimation pieuse» et amplificatio biblique}

Un passage de la troisième distinctio de la Josephina se prononoce sur le statut épistémologique de l'amplificatio biblique pratiquée par le poète :

notre méditation... n’affirme rien de façon téméraire

sur ce qui nous est inconnu, mais recourt modérément

aux conjectures par moyen d'un raisonnement topique.

À partir de ce qui est écrit, l'esprit, par un effort pieux,

16 Gerson, De laudibus elegie spiritualis (Quisquis amas), in : Fabre (2005) 455, v. $11-14$; Gerson, De laude musice (Musica divini), in : Fabre (2005) 448, v. 11-14.

17 Gerson, De laude musice (Musica divini), in : Fabre (2005) 450-451, v. $45-84$; Gerson, De laudibus elegie spiritualis (Quisquis amas), in : Fabre (2005) 455, v. 15-24. À ce sujet, voir Di Stefano (1971) 26-27.

18 Parmi la vaste littérature à ce sujet, voir Charland (1936) ; Bériou (1998); D’Avray (1985) ; Longère (1983) ; Briscoe -Jaye (1992).

19 Voir par exemple Alexandre de Halès, Summa theologiae, Tractatus introductorius, q. 1, a. 1, c. 4, ad 2 : ... modus definitivus debet esse, divisivus, collectivus, et talis modus debet esse in humanis scientiis, quia apprehensio veritatis secundum humanam rationem explicatur per divisiones, definitiones, et ratiocinationes.

20 Cicéron, De Inventione, I, 34, 57.

21 Pour la réception médiévale de ces oeuvres et la tradition des commentaires, voir Ward (2006) 3-75; Ward (1995).

22 Rhetorica ad Herennium, I.8, éd. T.E. Page et alii (1964), Cambridge, Mass., The Loeb Classical Library, 22-24:Id quod in negotionum expositione positum est tres habet partes : fabulam, historiam, argumentum. Fabula est quae neque veras neque veri similes continet res, ut eae sunt quae tragoediis traditae sunt. Historia est gesta res, sed ab aetatis nostrae memoria remota. Argumentum est ficta res quae tamen fieri potuit, velut argumenta comoediarum... (je souligne). 
est capable d'inférer ce qui n'est pas mis par écrit.

C'est ainsi qu'une foi certaine révèle ce qui est incertain,

quels ont été les faits ou ce qui aurait pu arriver ${ }^{23}$ (je souligne).

La conception de la poésie comme un récit fondé sur ce qui aurait pu arriver plutôt que sur des choses réellement survenues provient de la Poétique d'Aristote. À ce titre, le philosophe considère la poésie comme «plus philosophique et d'un caractère plus élevé que l'histoire ; car la poésie raconte plutôt le général, l'histoire le particulier. Le général, c'est-à-dire que telle ou telle sorte d'homme dira ou fera telles ou telles choses vraisemblablement ou nécessairement ${ }^{24}$ ». Le qualificatif de "philosophique» pour caractériser le procédé poétique renvoie à un principe aristotélicien contenu dans les Topiques ${ }^{25}$ : «si deux énoncés contradictoires ne peuvent être simultanément vrais, ils peuvent toutefois être simultanément probables». Philosophie et poétique convergent ainsi dans le terrain du raisonnement topique : de même que la philosophie se sert de prémisses probables et d'une argumentation basée sur les lieux (topoi) tout en prétendant être probante, de même la poésie aspire à une certaine forme d'universalité par moyen d'un raisonnement probable ou topique. Les deux démarches emploient des modalités d'argumentation qui se veulent crédibles et convaincantes, mais qui échappent aux exigences de la déduction formelle.

Autour des mêmes années, en 1416, Gerson traite plus systématiquement de cette question dans la Declaratio compendiosa quae veritates sint de necessitate salutis credendae. Ce traité se trouve à la base de la légitimation de la poésie comme moyen d'expression de la vérité doctrinale, en même temps qu'il établit les fondements épistémologiques de l'élaboration poétique. Dans une classification normative du discours doctrinal, la Declaratio établit six degrés (gradus) de vérité selon un ordre décroissant d'adhésion ${ }^{26}$. Les trois premiers correspondent à la doctrine orthodoxe telle qu'elle est explicitement contenue dans les Écritures ou transmise par la tradition apostolique. Ces vérités, directement révélées par Dieu, constituent les articles de la foi, nécessaires au salut. Les trois degrés suivants découlent logiquement des premiers, et ne détiennent qu'une valeur probable. Parmi ceux-ci, le plus bas degré correspond aux vérités qui servent à «édifier la charité ou la dévotion du cœur

23 Josephina, dist. III, v. 855-860 : Nil super ignotis igitur meditatio nostra/Affirmet temere, sola suffecerit uti/Coniecturarum thopica ratione modeste./Ex scriptis inferre potest non scripta pio mens/ Cum studio, sic certa fides incerta revelat/Qualia sunt acta vel que fieri potuere. Voir aussi les vers programmatiques à la fin du Prologue, v. 92-94 : ...favete/Nostraque sit, facite pietas accepta Camene,/Ancillans fidei, nulli preiudica vero,/Multa probabiliter suadens, temeraria nusquam,/Apta peregrinum cor sursum attollere celis. Voir aussi Gerson, sermon Jacob autem genuit, éd. Glorieux, $5.345-346$.

24 Poétique, c. 9, 1451a36-10. Au sujet de la vraisemblance poétique chez Aristote, voir Poétique, 1447a18-1448a27, 1448b4-23.

25 Aristote, Topica I, c. 10 et VIII, c. 11 ; voir aussi Auctoritates Aristotelis 36.121, éd. J. Hamesse (1974), Les "Auctoritates Aristotelis» : Un florilège médiéval, Louvain, 331 et 124.

26 Gerson, Declaratio compendiosa, éd. Glorieux, 6.181-189. 
pieux», plutôt qu'elles n'instruisent l'intellect. Ces vérités pieuses, l'Église autorise à croire $^{27}$. Elles résultent d'un jugement «estimatif» ou "croyance pieuse» (existimatio vel pia credulitas) qui échappe à la logique binaire du vrai et du faux, dans la mesure où elle relève du probable. En d'autres termes, une croyance pieuse est vraie si elle n'est pas entièrement improbable. Quant à la définition de' "estimation pieuse» comme forme de «raisonnement topique ou moral», elle s'inscrit dans la tradition des commentateurs de l'œuvre aristotélicienne qui élaborent une conception élargie du raisonnement logique pour inclure une stratégie de persuasion. Dans ce contexte, l'aestimatio dénote une forme de raisonnement à visée pratique ou morale, distinct et inférieur, du point de vue épistémique, du raisonnement démonstratif propre aux sciences spéculatives ${ }^{28}$.

La Josephina présente de nombreux exemples de ce genre d'agencement du récit biblique. Dans sa reconstruction de l'épisode de la fuite en Égypte, Gerson imagine qu'en y arrivant, Joseph aurait voulu dissimuler prudemment l'identité de son épouse. Il s'inspire ici d'un passage de la Genèse, qui attribue ce comportement à Abraham lors de son séjour en Égypte ${ }^{29}$. Un autre exemple est fourni par l'épisode des Rois mages, où l'absence de Joseph dans le récit évangélique est à la fois expliquée et comblée par une comparaison avec l'épisode de l'annonce aux bergers, où Joseph figure bel et bien aux côtés de la Vierge et l'enfant ${ }^{30}$.

27 Gerson, Declaratio compendiosa, éd. Glorieux, 6.184-185 : in sexto gradu... collocantur veritates illae quae tantummodo faciunt ad nutriendam vel fovendam devotionis religiosam pietatem, quae scilicet magis inducuntur ad inflammandum affectum quam ad instruendum intellectum, ubi pietas devota magis inspicitur quam veritas certa, ubi hoc unum reprobatur si adesset assertionis temeritas priusquam elucidaretur alio modo vel rationem certam ipsa veritas... Cadit existimatio vel pia credulitas non super veritate vel falsitate sed tantummodo super probabilitate vel apparentia... de talibus eligibilius est pie dubitare quam temere definire.

28 Voir aussi Quaestio in poetriam (XIII ${ }^{\mathrm{e}}$ siècle) anonyme, in : Dahan (1980) 171-247, surtout 216, où la notion de aestimatio est utilisée pour désigner l'assentiment qui correspond au champ de l'imaginaire poétique. À ce sujet, voir Marmo (1990) 145-198 ; voir aussi l'article plus récent de Robert (2012) 27-45.

29 Cf. Gn 12.11-13; Josephina, d. II, v. 411-424 : Vir prior alloquitur sponsam : «Cognosco decora/Quod sis, o domina; gens ista libidine fervens/Fedis urgetur stimulis; si sciverit uxor/Quod mea sis, mihi quid nisi mors, o Virgo, paratur/Atque pudicicie tibi discrimen, Pharaonis/Ducet ad aspectum mox te manus improba servi./Sors indigna nimis, nos o tibi quid faciemus,/Blande puer, fugimus neu te unum perdat Herodes;/Deserimus dulcesque lares, carosque propinquos./Ecce quod irruimus in tanta pericula soli/ Ignoti atque inopes, lingue ignari. Deus alme,/Nobis quid facere voluisti? Queso, Maria,/Filia dic mea sis, hoc etas credere verum/Suadebit, reliquum auxilio committo superno".

30 Cf. Mt 2.11 et Lc 2.16 ; Josephina, d. IX, v. 2278-2287: Invenisse Joseph pastores littera tradit,/Mentio nulla fit hic; sed qualiter huius honoris,/Huius mysterii mansisse putabitur exsors/Presertim reges quia non cessere repente/De (reditu) vetito, nam sompnis visio facta est./Quid? Quod muneribus erat aptus suscipiendis/Atque recondendis, an sola relicta videtur/Mater cum puero, quando vaga fama per urbem/Insonuit totam reges venisse potentes/In dromedariis cum pulchro et divite cultu. 


\section{Tyrannicide et herméneutique biblique}

D'autres lieux de la Josephina permettent d'illustrer, pour finir, les enjeux politiques des présupposés herméneutiques de Gerson. En guise d'épilogue à l'épisode du massacre des Innocents traité dans la dixième distinctio, le poète affirme que s'il est juste de tuer des tyrans sanguinaires, cela incombe seulement Dieu ou ses ministres (ministris) $^{31}$. L'allusion à l'actualité politique ne saurait pas nous échapper. Rappelons que le 23 novembre 1407, Louis d'Orléans, frère du roi Charles VI, est assassiné sur l'ordre de Jean sans Peur, duc de Bourgogne, son rival pour la régence. En mars de l'année suivante, Jean Petit, avocat à la solde de Jean sans Peur, prononce à Paris l'apologie du meurtre de Louis d'Orléans, connue comme la Justification du duc de Bourgogne. Le duc est ensuite acquitté, alors que la guerre civile ne tardera pas à éclater. Gerson réagit à maintes reprises contre la doctrine de Jean Petit à maintes reprises entre 1408 et $1413^{32}$. Après une première condamnation, éphémère, de la Justification du duc de Bourgogne en 1414, l'affaire Jean Petit occupa Gerson - en vain - pendant une bonne partie de son séjour à Constance.

Dans sa justification du tyrannicide, l'avocat de la cause bourguignonne soulève la question de l'interprétation du texte biblique, notamment le sixième commandement du Décalogue, «Tu ne tueras point». La Justification soutient que lorsqu'un sujet du roi porte atteinte ou complote contre sa personne, il est licite de le faire tuer comme un tyran traître et infidèle ${ }^{33}$. Jean Petit prétend ainsi que l'homicide compte comme «tyrannicide licite» lorsqu'il s'agit de protéger le roi d'un vassal qui complote sa destruction - c'est-à-dire, d'un vassal qui s'auto-condamne comme «tyran» selon la définition de Petit ${ }^{34}$.

Cherchant à dissoudre le conflit entre la thèse du tyrannicide et les lois $d u$ Décalogue, les défenseurs de Jean Petit à Constance prônaient une distinction herméneutique au sein du sens littéral entre une grammaire «basse», dont il fallait se méfier car subjective et trop attachée à la lettre, et une grammaire «haute», qui correspond à l'intention objective de l'auteur, le Saint-Esprit ${ }^{35}$. Gerson trouvait que ce

\footnotetext{
31 Josephina, dist. X, v. 2504-2507 : Cuilibet est ; si fas sevos jugulare tirannos/Cur sceleratus, cur truculentus vivit Herodes ?/Sed non fas; tibi vindictam, Deus alme, reservas./Solis quos statuis est exercenda ministris.

32 Voir par exemple Gerson, Veniat pax, éd. Glorieux, 7.2.1100 -1123 ; Id., Rex in sempiternum vive !, éd. Glorieux, 7.2.1005-1030.

33 Jean Petit, Justificatio ducis Burgundiae, tertia veritas, in : E. Du Pin (éd.), Ioannis Gersonii Doctoris Theologi et Cancellarii Parisiensis Opera Omnia, vol. 5, Anvers, 1706, col. 15-42, ici col. 27. 34 Jean Petit, Justificatio Ducis Burgundiae, col. 24.

35 Jean Petit, Justificatio ducis Burgundiae, col. 28-30 : in omni lege sunt duo. Primum, est sententia textualis. Aliud, est finis propter quem facta fuit lex, quem finem, conditores legis intendebant principaliter. Et quando accidit quod sententia textualis est contraria fini, debet epikë̈are lex ad intentionem finis, et non ad sensum litteralem. À ce sujet, on consultera Froehlich (1977) 20-48; Coville (1932).
} 
dédoublement du sens littéral était indésirable, car il pouvait semer la confusion et affaiblir l'autorité et du texte biblique et des arguments théologiques qui en dérivent. Selon le chancelier, il n'y a qu'un sensus litteralis, critère ultime d'interprétation biblique, qui résulte de l'intention de l'auteur des Écritures et que seule l'Église est en mesure de circonscrire contre l'arbitraire individuel ${ }^{36}$. Gerson s'inspire ici des règles herméneutiques du De doctrina christiana d'Augustin, en particulier la distinction entre la lettre du texte (la signification sémantique), et l'intention de l'auteur cachée derrière les mots dont il se sert (la signification dianoétique). Selon Augustin, l'interprète qui assujettit son regard à la lettre fait une lecture selon la chair (carnalis, corporalis), contrairement à l'ordre naturel qui prescrit la subordination du corps à l'esprit. Celui-ci se sert des réalités matérielles pour les dépasser vers une signification supérieure ${ }^{37}$.

Toujours est-il que, suivant un trait caractéristique de l'exégèse tardo-mediéva$1 \mathrm{e}^{38}$, Gerson développe une distinction entre la lettre et le sens littéral de la Bible, articulée en termes d'une opposition entre le sensus litteralis, l'intention de l'auteur, et le sensus logicalis, le sens grammatical du texte. Il importait donc d'élucider cet éclatement apparent du sens littéral sans conforter les principes herméneutiques de Jean Petit, en particulier son instrumentalisation de II Cor. 3, 6 : littera enim occidit, pour relativiser la normativité de la sixième loi du Décalogue, «Tu ne tueras point» ${ }^{39}$. C'est la notion buridanienne d'une «double logique ${ }^{40}$ qui permettra à Gerson de sortir de l'écueil : la lettre biblique n'est pas soumise aux critères de la logique spéculative, mais suit plutôt les règles de la rhétorique, plus adaptée au langage figuré des Écritures et sensible au contexte dans l'explication de la signification des mots. Car «l’Écriture sacrée dans sa qualité de science morale et historique (histo-

36 Gerson, De sensu litterali sacrae Scripturae, éd. Glorieux, 3.335 : Tertia [propositio] : sensus Scripturae litteralis iudicandus est prout Ecclesia, Spiritu sancto inspirata et gubernata, determinavit et non ad cuiuslibet arbitrium et interpretationem.

37 Augustin, De doctrina christiana I.36.41 ; III.10.15 ; Id., De Gen. ad litt., I.2.5 ; Id., De util. cred. 3.5. À ce sujet, Eden (1990) 45-63.

38 Voir Froehlich (1977) 46-47.

39 L'adage Semper tenere litteralem sensum in sacra scriptura est occidere animam suam est une paraphrase du caveat qu'Augustin énonce dans le De doctrina christiana, III.5.9. Pour l'emploi du verset paulinien par Jean Petit, voir Justificatio ducis Burgundiae, col. 29-30 ; voir aussi Chartularium Universitatis Parisiensis, IV, 278, n. 2011, Octava assercio ; Gerson, Errores circa praeceptum : Non occides, éd. Glorieux, 10.273-274 ; Id., Réponse à la consultation des maîtres (Constance, 4 décembre 1415), éd. Glorieux, 10.233. Au sujet des diverses interprétations du passage paulinien, voir Froehlich (1977), 28-29 ; Dahan (2009) 237-262, surtout 258-260.

40 Voir Jean Buridan, Questiones in rhetoricam Aristotelis, I.2 ; Id., Quaestiones super decem libros Ethicorum, Paris, 1513 (réimpr. Frankfurt a.M., 1968), f. 2a-b. Buridan s'inspire d'Aristote, Ethique à Niomaque, I, ch. 1, 1094b 11. Voir aussi Gerson, lettre Jucundum est aux Messieurs de Navarre (1400), éd. Glorieux, 2.32 ; Id., De duplici logica, éd. Glorieux, 3.57-63. Sur l'origine buridanienne de la conception de la rhétorique comme une logique propre aux «sciences morales», voir Kaluza (1994) 197-258, en part. $233-238$. 
rialis, narrative) possède sa propre logique, qu'on appelle la rhétorique ${ }^{41} »$. La rhétorique est donc pour ainsi dire la façon dont Dieu nous parle dans la Bible, qu'il faut distinguer de la surface grammaticale du texte. Elle suit ses propres critères d'interprétation, qui relèvent davantage de l'intellect pratique ou affectif que de l'intellect spéculatif. Dans cette optique, le sens littéral induit en erreur seulement lorsqu'il est l'objet d'une interprétation suivant des critères de vérité issus de la logique spéculative. En tant que science morale, l'Écriture condescend à l'usage commun de la langue et se sert de figures et de tropes afin d'attiser l'affect des auditeurs.

Comme le signale à juste titre Zach Flanagin ${ }^{42}$, la distinction entre la lettre (gouvernée par la logique spéculative) et le sens littéral (obéissant aux règles de la rhétorique) introduite par Gerson vise moins à établir un double référent historique qu'à promouvoir l'idée selon laquelle la Bible est un texte mystique qui cèle une vérité spirituelle. À ce sujet, Gerson récupère et adapte une vieille tradition victori$\mathrm{ne}^{43}$, selon laquelle la surface grammaticale du texte biblique sert de porte d'entrée à une réalité supérieure ${ }^{44}$. S'il conçoit la Bible comme un texte mystique dont la lecture entend nous ramener à Dieu, cette signification supérieure du texte biblique, contrairement aux victorins, ne correspond pas au sens spirituel. On l'a vu, l'herméneutique biblique de Gerson nuance la distinction entre l'esprit et la lettre pour accueillir deux niveaux de littera : le scriptum et l'intention de l'auteur. La signification supérieure du texte biblique est donc plutôt considérée comme faisant partie du sens littéral de la Bible. Afin d'articuler cette double signification du sens littéral, Gerson recourt à la distinction psychologique des facultés de l'âme entre volonté et intellect. Ainsi, l'Esprit peut communiquer par moyen de la signification première des mots (termini) dans le but d'éveiller l'affect et de susciter l'action. Il nous parle ainsi lorsqu'il s'agit du contenu moral ou historique des Écritures. Ici, la signification première de la lettre suffit pour nous transmettre l'intention de l'auteur. À un second niveau, la signification littérale entend stimuler notre intellect vers des réalités supérieures. Tout comme le premier se sert des mots pour inciter l'affect, ce second niveau utilise des choses (res) qui représentent à leur tour d'autres réalités. Il s'agit ici du sens littéral, par opposition au sens grammatical de la lettre. C'est seulement à ce deuxième niveau «intellectif» que l'on peut dire à juste titre que «la lettre tue» ${ }^{45}$.

41 Gerson, De sensu litterali s. Script., prop. 2, éd. Glorieux, 3.334 : Sensus litteralis sacrae Scripturae accipiendus non est secundum vim logicae seu dialecticae, sed potius iuxta locutiones in rhetoricis sermonibus usitatas, et iuxta topos et figuratas locutiones quas communis usus committit, cum consideratione circumstantiarum litterae ex praecedentibus et posterius appositis. Habet enim Scriptura sacra, sicut et moralis et historialis scientia, suam logicam propriam, quam rhetoricam appellamus. 42 Flanagin (2006) 133-177, surtout p. 154-159.

43 Voir Hugues de Saint-Victor, Didascalicon, c. 3, éd. Buttimer, Washington, 1939, 116.

44 Hugues de Saint-Victor, De scripturis et scriptoribus sacris, 14 (PL 175), col. 20 - 21.

45 Gerson, Réponse à la consultation des maîtres, éd. Glorieux, 10.239-240 ; Id., De examinatione doctrinarum, éd. Glorieux, 9.463 : Unde generaliter in parabolicis locutionibus sensus litteralis est, non qui per verba, sed per res et facta designatur, ut de lignis constituentibus sibi regem [Jg. 9.8], etc. 
Et qu'une considération des circonstances de la lettre et des figures rhétoriques peut s'avérer féconde pour l'intellect qui cherche une connaissance spirituelle. La Josephina combine à son tour ces deux niveaux de signification littérale : l'histoire de Joseph et Marie doit être lue dans un sens à la fois littéralement prescriptif (leurs mœurs et leur piété sont à imiter), et comme conduisant à une réalité supérieure (leur mariage virginal symbolise l'union mystique de l'âme à Dieu).

\section{Conclusion}

Les considérations herméneutiques de Gerson reflètent la limite très floue entre sens littéral et sens spirituel qui caractérise l'exégèse Moyen Âge tardif, dans la mesure où la pluralité des sens spirituels semble être absorbée par le «vrai» sens littéral, désigné par l'intention de l'auteur divin ${ }^{46}$. La chose se complique davantage avec les enjeux propres au schisme. Car Gerson admet un troisième niveau interprétatif, celui de la tradition extratextuelle transmise par la succession apostolique, incluant les écrits des Pères et des docteurs de l’Église. Lorsque le chancelier fait référence à la «tradition» comme norme interprétative, c'est surtout pour souligner l'importance de se tenir à un certain stylus theologicus, dans le sens de la manière courante de parler (usus loquendi) des docteurs et des anciens interprètes des Écritures ${ }^{47}$. Ici, le clivage herméneutique entre la lettre et l'esprit obtient en termes de «sens théologique littéral» (sensus theologicus litteralis), par opposition au «sens logique» grammatical (sensus logicalis). Selon Gerson, le sens théologique littéral est le vrai sens de la Bible, qui correspond à l'interprétation transmise par l'Église depuis ses origines apostoliques et à travers certaines révélations ${ }^{48}$. Dans cette optique, la Josephina serait un exemple emblématique de la mise en application des principes herméneutiques de Gerson : un récit gouverné par une interprétation «théologique littérale» des implicites bibliques, là où le théologien-poète limite son intervention à une explicitation doctrinale, guidée par la rhétorique, de ce qu'il estime être l'intention de l'auteur divin.

46 À ce sujet, voir Froehlich (1977), 45 - 46, qui souligne l'importance donnée au sens littéral comme un trait saillant de l'exégèse de la fin du Moyen Âge ; voir aussi Smalley (1952) 69 ; Dahan (2009) 237262 ; Dahan (2014) 115-142, surtout 128-129.

47 Gerson, Réponse à la consultation des maîtres, éd. Glorieux, 10.241. À ce sujet, voir Burrows (1991) 88-125 ; Iribarren (2017) 183-221.

48 Voir Gerson, De sensu litterali Sacrae Scripturae, sexta prop., éd. Glorieux, 3.335 ; Id., Declaratio compendiosa, 6.182 ; Id., De necessaria communione laicorum sub utraque specie, regula 9, éd. Glorieux, 10.58 . 


\section{Bibliographie}

Bataillon (1982): Louis J. Bataillon, «Intermédiaires entre les traités de morale pratique et les sermons: les distinctiones bibliques alphabétiques», in: Les genres littéraires dans les sources théologiques et philosophiques médiévales, Louvain, 213-226.

Bataillon (1993): Louis J. Bataillon, La prédication au XIIIe siècle en France et en Italie, Aldershot.

Bériou (1998): Nicole Bériou, L'avènement des maîtres de la parole. La prédication à Paris au XIII' siècle, 2 vols, Paris.

Briscoe - Jaye (1992): Marianne G. Briscoe et Barbara H. Jaye, Artes praedicandi and Artes orandi, Turnhout.

Burrows (1991): Mark Burrows, Jean Gerson and De Consolatione Theologiae (1418). The Consolation of a Biblical and Reforming Theology for a Disordered Age, Tübingen.

Charland (1936): Thomas-Marie Charland, Artes Praedicandi. Contribution à l'histoire de la rhétorique au Moyen Âge, Paris-Ottawa.

Chenu (1964): Marie-Dominique Chenu, "La décadence de l'allégorisation. Un témoin: Garnier de Rochefort (v. 1200)», in: L'homme devant Dieu. Mélanges offerts au P. Henri de Lubac, t. II, Paris.

Combes (1964): André Combes, La théologie mystique de Gerson. Profil de son évolution, 2, Rome.

Coville (1932): Alfred Coville, Jean Petit: La question du tyrannicide au commencement $d u X V^{e}$ siècle, Paris.

Dahan (1980): Gilbert Dahan (éd.), «Notes et textes sur la Poétique au Moyen Âge», in: Archives d'histoire doctrinale et littéraire du Moyen Âge, 47, p. 171-247.

Dahan (1999): Gilbert Dahan, L'Exégèse chrétienne de la Bible en Occident médiéval, XII ${ }^{e}$-XIV siècle, Paris.

Dahan (2009): Gilbert Dahan, «Le sens littéral dans l'exégèse chrétienne de la Bible au Moyen Âge», in: 0.-Th. Venard (dir.), Le sens littéral des Écritures, Paris, 237-262.

Dahan (2014): Gilbert Dahan, «Herméneutique et procédures de l'exégèse monastique ", in: G. Dahan et A. Noblesse-Rocher (dir.), L'exégèse monastique au Moyen Âge (Xle-XIV siècle), Paris, 115-142.

D'Avray (1985): David D'Avray, The Preaching of the Friars. Sermons diffused from Paris before 1300. Oxford.

Di Stefano (1971): G. Di Stefano, «II Trecento », in: C. Pellegrini (dir.), Il Boccaccio nella cultura francese, Atti del Convegno di studi "L'opera del Boccaccio nella cultura francese ", Certaldo 2-6 settembre 1968, Florence, 1- 47.

Dordoni (1996): Annarosa Dordoni, «Per la storia della devozione a san Giuseppe: indicazioni di metodo e linee di ricerca», in: Annali di Scienze Religiose 1, 321-342.

Du Pin (1706): Ellies Du Pin (éd.), Ioannis Gersonii Doctoris Theologi et Cancellarii Parisiensis Opera Omnia, Anvers.

Eden (1990): Kathy Eden, «The Rhetorical Tradition and Augustinian Hermeneutics in De doctrina Christiana», in: Rhetorica, 8/1, 45-63.

Fabre (2006): F. Fabre, «Pétrarque poète chrétien ? La critique de l'Africa par Jean Gerson», in: E. Duperray (dir.), La postérité répond à Pétrarque. Sept siècles de fortune pétrarquienne en France., Paris, 111-125.

Fabre (2005): Isabelle Fabre (éd.), La doctrine du chant du cœur de Jean Gerson. Édition critique, traduction et commentaire du "Tractatus de canticis » et du "Canticordum au pèlerin », Genève.

Fabre (2011): Isabelle Fabre, «Un hault chant de unisson en souveraine silence: étapes et contours de l'ascension mystique dans le Canticordum au Pelerin de Jean Gerson (1363-1429)», in: S. 
Triaire et P. Victorin (dir.), Deviser, diviser. Pratiques du découpage et poétiques du chapitre de l'Antiquité à nos jours, Montpellier, 79-107.

Flanagin (2006): Zach Flanagin, «Making sense of it all: Gerson's biblical theology», in: Brian P. McGuire (dir.), A Companion to Jean Gerson, Leiden-Boston, 133-177.

Froehlich (1977): Karlfield Froehlich, «»Always to Keep the Literal Sense in Holy Scripture Means to Kill One's Soul«: The State of Biblical Hermeneutics at the Beginning of the Fifteenth Century ", in: Earl Miner (dir.), Literary Uses of Typology, from the Late Middle Ages to the Present, Princeton, $20-48$.

Glorieux (1962-1973): Palémon Glorieux (éd.), Jean Gerson. Oeuvres complètes, Paris-Tournai. Hamesse (1974): Jaqueline Hamesse (éd.), Les «Auctoritates Aristotelis»: Un florilège médiéval, Louvain.

Hasenohr (1978): Geneviève Hasenohr, «Un recueil de distinctiones bilingue au début du XIV $s$ », in: Romania 99, 46-96 et 183-206.

Hobbins (2009): Daniel Hobbins, Authorship and Publicity before Print. Jean Gerson and the Transformation of Late Medieval Learning, Pennsylvania.

Iribarren (2017a): Isabel Iribarren, «Réception et fonctions des récits apocryphes dans la Josephina de Jean Gerson», in: Apocrypha 28, p. 187-229.

Iribarren (2017b): Isabel Iribarren, «Question de style. Langage et méthode comme enjeux rhétoriques dans l'œuvre de Gerson», in: U. Zahnd (dir.), Langage et méthode. Réflexions historiques et historiographiques sur la pensée médiévale, Freiburg-Bg, 183-221.

Iribarren -Roccati (2019): Isabel Iribarren (trad., introd., notes et commentaire) et G. Matteo Roccati (édition critique), Josephina. L'épopée de saint Joseph, Paris.

Kaluza (1994): Zenon Kaluza, «Les sciences et leurs langages. Note sur le statut du 29 décembre 1340 et le prétendu statut perdu contre Ockham », in: L. Bianchi (dir.), Filosofia e teologia nel trecento, Louvain-la-Neuve, 1994, 197-258.

Landgraf (1973): Artur M. Landgraf, Introduction à l'histoire de la littérature théologique de la scolastique naissante, éd. française de A.-M. Landry, Montréal-Paris.

Lieberman (1955): Max Lierberman, «Chronologie gersonienne. IV: Gerson poète», in: Romania 76, $289-333$.

Longère (1983): Jean Longère, La prédication médiévale. Paris.

Marmo (1990): Costantino Marmo, "Suspicio, a Key Word to the Significance of Aristotle's Rhetoric in XIIIth Century Scholasticism », in: Cahiers de l'Institut du moyen âge grec et latin 60, 145-198.

Ouy (1962): Gilbert Ouy, « La plus ancienne œuvre retrouvée de Jean Gerson: le brouillon inachevé d’un traité contre Juan de Monzón (1389-1390)», in: Romania 83, 433-492.

Parkes (1976): Malcom B. Parkes, «The Influence of the Concepts of Ordinatio and Compilatio on the Delopment of the Book», in: J. J. G. Alexander et M. T. Gibson (dir.), Medieval Learning and Literature, Essays presented to Richard William Hunt, Oxford, 115-141.

Robert (2012): Aurelien Robert, "L'idée de logique morale aux XIII et XIV siècles», in: I. Costa, A. Robert et N. Bouloux (dir.), Philosophies morales. L'éthique à la croisée des savoirs (XIIle-XIVe siècles), Médiévales 63, 27-45.

Roccati (1980): G. Matteo Roccati (éd.), J. Gerson, Oeuvre poétique latine, thèse de troisième cycle, Paris, EHESS.

Roccati (1982): G. Matteo Roccati, «Gerson e il problema dell'espressione poetica: note su alcuni temi e immagini ricorrenti nelle poesie latine», in: Studi francesi 77, 278-285.

Roccati (2001): G. Matteo Roccati (éd.), Jean Gerson. Josephina, Paris, LAMOP (CD-ROM).

Rouse (1974): Richard H. et Marie A. Rouse, "Biblical Distinctions in the XIIlth century», in: Archives d'histoire doctrinale et littéraire du Moyen Âge 41, 27-37.

Seitz (1908): Joseph Seitz, Die Verehrung des hl. Joseph in ihrer geschichtlichen Entwicklung bis zum Konzil von Trient dargestellt, Freiburg. 
Smalley (1952): Beryl Smalley, The Bible in the Middle Ages, Oxford.

Ward (1995): John O. Ward, Ciceronian Rhetoric in Treatise, Scholia and Commentary, Turnhout. Ward (2006): John O. Ward, «The Medieval and Early Renaissance Study of Cicero's De inventione and the Rhetorica ad Herennium. Commentaries and Contexts", in: V. Cox et J. O. Ward (dir.), The Rhetoric of Cicero in Its Medieval and Renaissance Commentary, Leiden, 3-75.

Weijers (1991): Olga Weijers, Dictionnaires et répertoires au Moyen Âge. Une étude de vocabulaire, Turnhout.

Wilmart (1940): André Wilmart, «Un répertoire d’exégèse composé en Angleterre vers le début du XIII ${ }^{\text {e }}$ siècle», in: Mémorial Lagrange, Paris, 307-346.

Witt (1977): Ronald Witt, "Coluccio Salutati and the Conception of the Poeta Theologus in the Fourteenth Century», in: Renaissance Quarterly 30, 538-563.

Zink (2003): Michel Zink, Poésie et conversion au Moyen Âge, Paris. 
